\title{
PRIMARY PULMONARY HYPERTENSION
}

\author{
BY \\ W. D. BRINTON \\ From Guy's Hospital
}

Received December 20, 1949

Primary pulmonary vascular sclerosis was first fully described by Brenner (1935) who stressed the presence of hypertrophy of the right, but not of the left ventricle; he concluded his description with the opinion that primary sclerosis is not a pathological entity but includes several conditions. Other authors, notably De Navasquez et al. (1940), preferred the term " primary or idiopathic right ventricular hypertrophy." Until recently pulmonary hypertension was an assumption from the physical signs and morbid anatomy, but in the last few years evidence has accrued from cardiac catheterization that it does in fact exist in these cases.

By definition, in primary pulmonary hypertension, none of the known causes of pulmonary hypertension are present: it is therefore not surprising that few suggestions as to the cause are reported. The only experimental evidence available is that of Harrison (1948) who, working on rabbits, produced lesions in the smaller pulmonary arteries morphologically indistinguishable from spontaneous arteriosclerosis, by means of intravenous injections of finely fragmented fibrin. He was unable to produce significant hypertrophy of the right ventricle.

The purpose of this paper is to record a possible cause for some of these cases.

\section{CASE Notes}

S.B., aged 26, was admitted to Guy's Hospital under Dr. Mann on February 23, 1949, complaining of shortness of breath for 18 months and loss of voice for one week.

He had a normal childhood and worked as a labourer until he joined the army in 1939 . In 1945, in India, he had dysentery; he was in hospital for 6 weeks and was given tablets, but no injections, and made a good recovery. In 1946, in Burma, he was admitted to hospital complaining of right-sided pain, first thought to be due to amœbic hepatitis but later considered to be due to pleurisy, probably of tuberculous origin. There was fever for 10 days without leucocytosis and the pain spread to the left chest. On one occasion fine crepitations were heard at both lung-bases and a chest.X-ray showed "tenting" of the right diaphragm with small areas of infiltration at both bases. At no time was a rub heard and there was no effusion. Four days after the first X-ray, a second film showed that the infiltration had gone; and by now he was apyrexial and feeling well. However, he was sent home by hospital ship as a case of probable tuberculous pleurisy. On his arrival in this country he was noted to be perfectly well and to have no complaints; no physical signs of disease were found. The diagnosis of tuberculosis was abandoned therefore, and he was regraded in medical category C2, but kept in the army. In his army notes there is no mention of dyspnca, of anæmia, or of any cardiovascular abnormality, but when later the patient observed the interest taken in his cardiac signs by those attending him at Guy's he stated that the army doctors had shown the same concern.

He was demobilized early in 1947 and worked as a labourer until October of that year when he began to notice shortness of breath. He went to the Royal Berkshire Infirmary, Reading, and was admitted. He was found to be pale $(\mathrm{Hb} .70 \%)$ and his heart was enlarged. His blood pressure was $120 / 60$. There were no signs of disease in the chest. Occult blood was found in the stools but 
a barium meal showed no abnormality of œsophagus, stomach, or duodenum. An X-ray report at that time stated: "Heart mitral in shape with prominent hilar vascular shadows; the bases are clear."

He discharged himself after three weeks in hospital, but since that time his breathlessness on exertion had become progressively worse, so that for the last five months he had been unable to walk more than 100 yards without becoming acutely short of breath. There had been no pleuritic pain since 1947 and no cough, but he had recently experienced a dull retrosternal pain when short of breath.

A week before admission he developed a cold in the nose and sore throat with fever and a slightly productive cough. Later that day he suddenly lost his voice and complained of retrosternal dysphagia. The fever and cough had continued since, with mild pleuritic pain on the right side.

On admission he stated that for the last 18 months he had had severe hæmorrhage from piles, losing up to half a cupful of blood daily. His weight had been 13 stone in 1947 (in the army it was noted that he was somewhat obese); and it was now $10 \frac{1}{2}$ stone. He did not smoke or drink heavily.

On examination he was very pale and dyspnœic at rest. There was no cyanosis, no clubbing of fingers, and no glandular enlargement. His temperature was $99.0^{\circ} \mathrm{F}$. The pulse was 120 , regular with a few extrasystoles. The blood pressure was 110/60. The apex-beat was forceful and diffuse but was not outside the mid-clavicular line. There was a marked systolic heaving pulsation in the second and third left intercostal spaces alongside the sternum and there was a palpable second sound here. This region was dull to percussion. On auscultation there was a harsh systolic murmur at the apex and an even louder systolic murmur over the heaving pulsation at the base, where the second sound was extremely loud. There was a mid-diastolic added sound, audible all over the heart but loudest at a point just medial to the apex. There was no diastolic murmur. There was no filling of the veins of the neck and no odema of the ankles. Laryngoscopy showed complete paralysis of the left vocal cord. In the chest, there was diminished air-entry and fine crepitations in the right axillary region. The liver was tender and enlarged to two fingers' breadth below the costal margin. There was no ascites. Rectal examination caused severe pain and spasm; no abnormality was felt, however, and there was no blood on the finger-stall.

The urine was normal throughout his illness. His hæmoglobin was 46 per cent with red cells 3.3 million. The white cells remained normal throughout. The chest X-ray showed slight cardiac enlargement (cardiothoracic ratio, $16 / 30 \mathrm{~cm}$.), and dilatation of the pulmonary artery and its main branches (Fig. 1). There was no abnormality in the lung-fields. The electrocardiogram showed marked right axis deviation with flat T II and deeply inverted T III, due to right ventricular strain (Fig. 2). The Wassermann and Kahn reactions were negative.

Clinical course. He was transfused with a pint of fresh blood, but during the next few days he continued to run an intermittent fever up to $101^{\circ} \mathrm{F}$. Blood cultures were sterile throughout his illness and examination of the sputum showed no abnormality. On the fourth day of admission he was started on penicillin. On the seventh day he had a sudden rigor, with temperature rising to $103^{\circ} \mathrm{F}$. and pleuritic pain in the right chest. He became acutely ill with cyanosis of lips and fingertips and respirations at $40 \mathrm{a}$ minute. He was placed in an oxygen tent and rallied somewhat. The next day he had another severe attack of air-hunger, this time with filling of the neck veins, and lumbar œdema was noted. Meanwhile the signs in his chest were unchanged. A further transfusion increased the signs of congestion. From then till his death on the tenth day after admission his temperature was subnormal. His general condition deteriorated, his air-hunger became continuous and was no longer relieved by oxygen. During the last four days he complained of severe pain beneath the sternum. He died in great distress, fighting for breath.

Clinical comment. On admission the signs of increased pressure and blood flow in the pulmonary artery suggested a diagnosis of auricular septal defect. The anæmia was considered to be due to chronic hæmorrhage from piles. There was no clinical evidence of mitral stenosis but he was always too ill to screen. Later, when the fever demanded attention, the possibility of a 
subacute bacterial endocarditis had to be considered. The pleuritic pain was thought to be due to infarction, either from local thrombosis or by emboli if there were vegetations upon the defect. He had indeed a previous history of some inflammatory disease of the chest, but this seemed altogether too trivial to account for his symptoms by obliteration of the pulmonary vascular bed. Further, there was no clinical evidence of emphysema.

The great dilatation of the main pulmonary artery, which was the outstanding physical sign in this case, was taken to be the cause of the left cord paralysis by tensiontraction on the recurrent laryngeal nerve. I have not been able to find a report of this complication in any previous case of primary pulmonary hypertension, but it has been described in cases of auricular septal defect by Erlanger and Levine (1943). In an interesting paper in which they add two further cases, one of mitral stenosis and the other a probable auricular septal defect, Dolowitz and Lewis (1948) support the hypothesis of Kraus (1901), that the process begins with enlargement of a strategically placed lymphnode with fixation of the left recurrent laryngeal nerve. After this fixation, enlargement of the aorta, of the pulmonary artery, or of the left auricle, could stretch and thus paralyse the nerve.

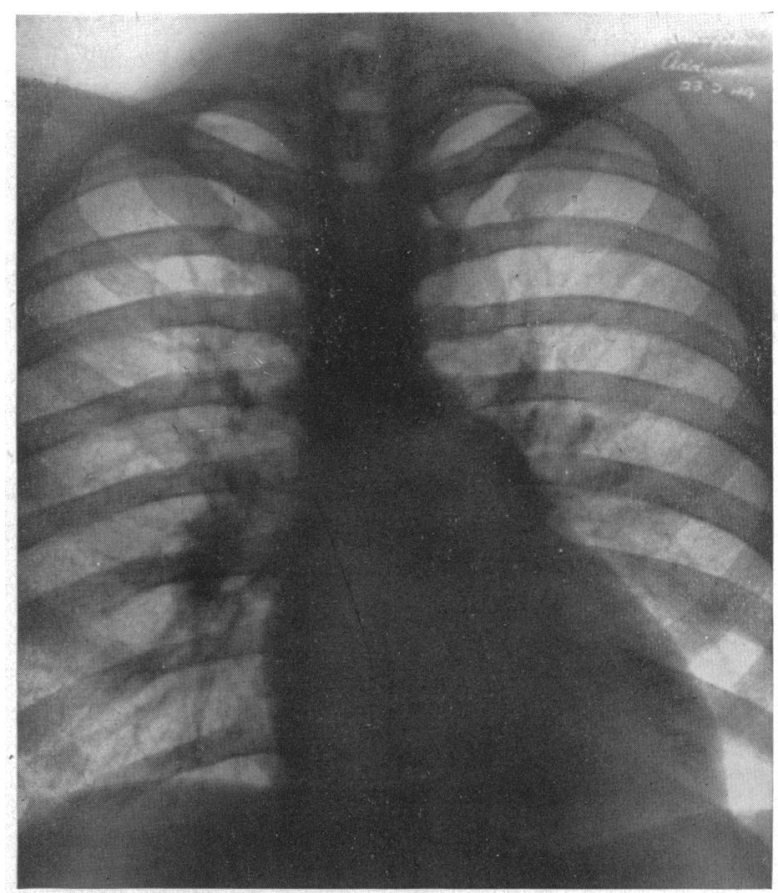

FIG. 1.-Teleradiograms of the chest, showing slight enlargement of the heart (cardiothoracic ratio $16: 30$ ) with prominent pulmonary conus and dilatation of main branches of the pulmonary artery.

During the course of this patient's illness, a pulmonary diastolic murmur was daily expected and carefully listened for. The dilatation of the pulmonary artery was fully sufficient to cause incompetence of the valve, but the murmur of regurgitation was never heard.

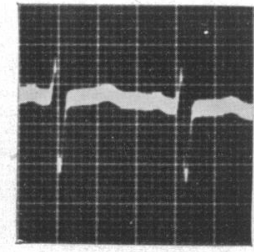

I.

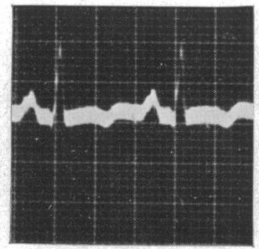

II.

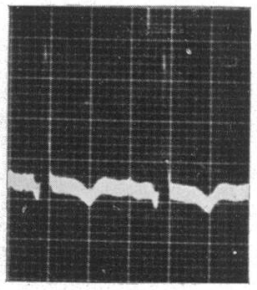

III.

FIG. 2.-Electrocardiogram, showing right axis deviation with flat TII and inverted TIII indicating right ventricular strain. V leads were not taken.

\section{POST-MORTEM FINDINGS}

The necropsy was performed by Dr. S. J. De Navasquez, about 10 hours after death. Only the relevant findings are given.

Cardiovascular system. The heart weighed $480 \mathrm{~g}$. , and of this the right ventricle weighed $143 \mathrm{~g}$. , the left ventricle $148 \mathrm{~g}$. This gives a left to right ratio of 0.96 against the normal 1.79 (Lewis, 1914). The heart was greatly enlarged by dilatation of all its chambers, particularly those on the right. 
The right ventricle showed great hypertrophy of the myocardium which measured $11 \mathrm{~mm}$. half-way to the apex. The tricuspid ring was dilated $(15 \mathrm{~cm}$. circumference) and so was the pulmonary ring $(9 \mathrm{~cm}$.). The mitral ring was much dilated $(12 \mathrm{~cm}$.) but the aortic ring $(6.5 \mathrm{~cm}$.) was normal. The endocardium was normal throughout, as were all the valves and there were no developmental abnormalities. The pulmonary artery was uniformly distended and showed patches of atheroma and mural thrombosis. Its main and medium-sized branches were dilated but normal. The aorta appeared to be smaller than normal, both compared with the pulmonary artery and with the general body build.

Respiratory system. The larynx was normal but the left vocal cord was nearer the mid-line than the right. Both pleural cavities were obliterated by old pale fibrous adhesions. The lungs were pale and œdematous but there was no evidence of inflammatory disease or infarction. The tracheobronchial lymph-glands were normal. .

Abdominal viscera. The peritoneal cavity contained $200 \mathrm{ml}$. of clear yellow fluid. The liver (2045 g.) was moderately enlarged with changes typical of passive congestion.

Microscopical. The right ventricle showed hypertrophy of the myocardial cells. The left ventricle was normal. Interest was centred on the pulmonary vasculature.

Sections from the hilum of each lung showed atheroma and mural thrombosis of the main branches of the pulmonary artery, which diminished peripherally till in vessels of about $3 \mathrm{~mm}$. diameter it was no longer seen. The smaller vessels were dilated but otherwise normal. A section from the periphery of the lung showed what appeared to be two small bronchial arteries, the lumen of which was obliterated by intimal thickening (see Fig. 3).

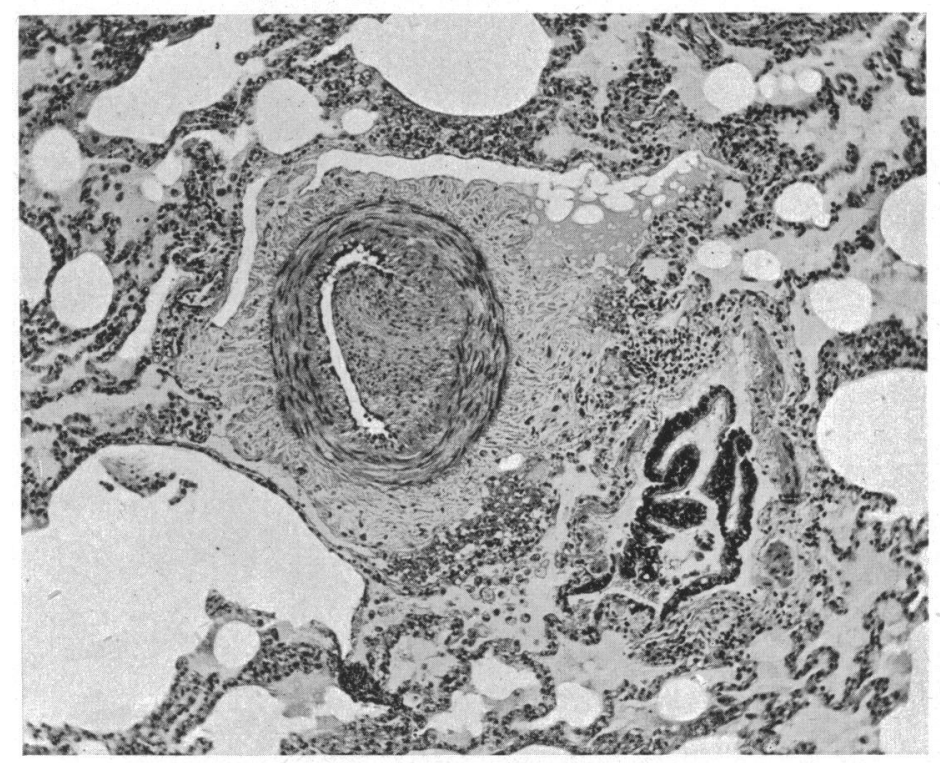

FIG. 3.-Photomicrograph from the periphery of the right lung, showing a medium-sized artery, the lumen of which is greatly narrowed by what appears to be an organized thrombus. H and E. Magnification, $\times 75$.

A section of the right lung taken from the hilum showed two separate conglomerations of entangled blood-vessels which consisted of arteries and veins communicating with each other, forming complicated arteriovenous aneurysms (Fig. 4 and 5). Both these aneurysms lay close to large bronchi. Serial sections of the entire block made to follow the course of these aneurysms showed that they eventually resolved into a large artery and a large vein with an intercommunicating lumen. Although it was impossible to identify the anatomy of these blood vessels with certainty, 


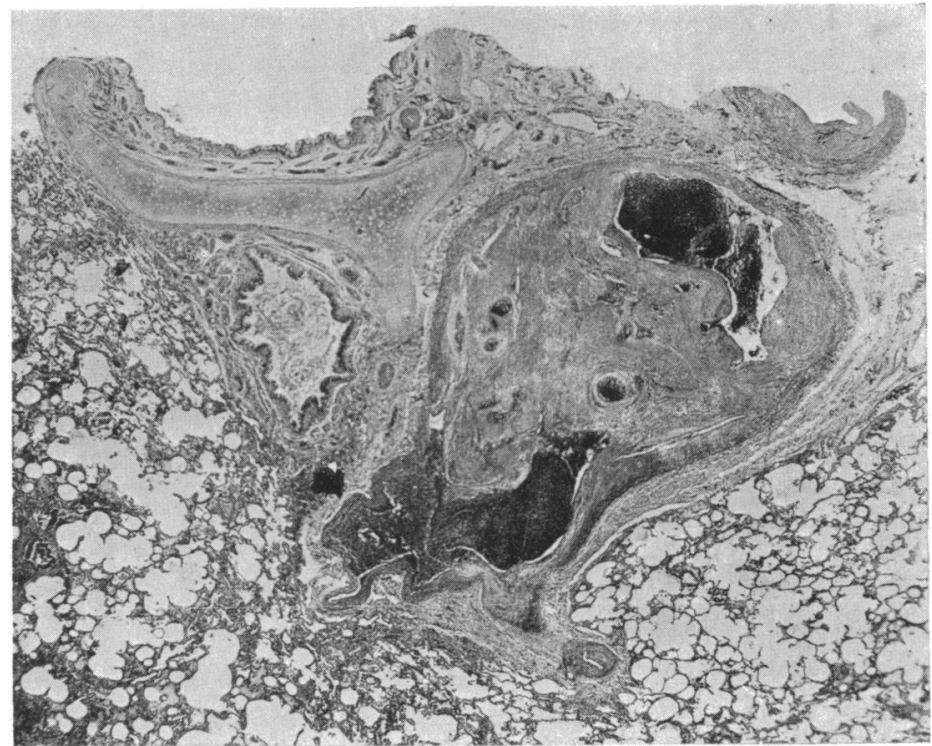

FIG. 4.-Photomicrograph of hilum of right lung, showing mass of intercommunicating blood-vessels by the side of a bronchus. $\mathrm{H}$ and $\mathrm{E}$. Magnification, $\times 12$.

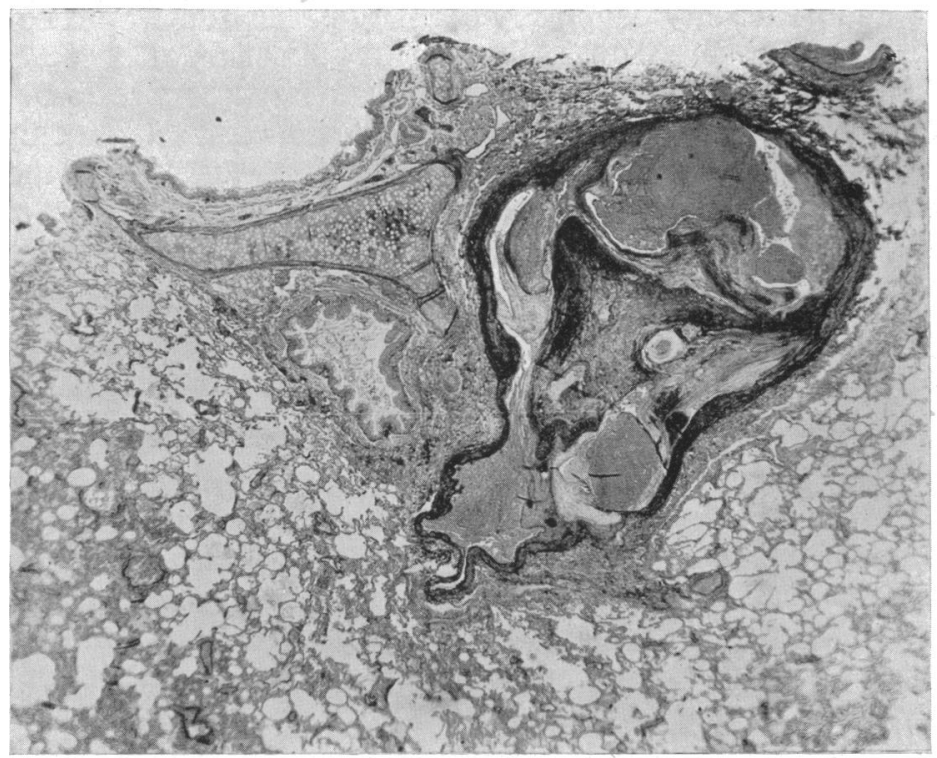

FIG. 5.-Photomicrograph from the same block stained by Weigert's elastic stain, showing abundant elastic tissue in the walls of the communicating vessels. Weigert's stain. Magnification, $\times 12$.

the morphology of the walls with their relative difference in the elastic and muscular components, suggested that the malformations were between bronchial arteries and pulmonary arteries and veins.

Sections from the lung showed moderate passive congestion with odema and heart-failure cells in the alveoli. Another section from the periphery showed moderate emphysema and focal œdema. There was no evidence of inflammatory disease or of infarction. 


\section{Discussion}

Can this case be accepted as one of primary pulmonary hypertension? It is suggested that the term is justified by the isolated right ventricular hypertrophy, with dilatation and early sclerosis of the pulmonary ariery without adequate pulmonary disease. Must the arteriovenous aneurysm found at the right hilum be regarded as causally related to the patient's illness? Here it must be admitted that the existence of so unusual an abnormality of the lung, in a patient who has died of a rare disease of the pulmonary vasculature of unknown ætiology, justifies this assumption as a working hypothesis.

Before death the diagnosis of auricular septal defect was made with some confidence, from the signs of increased blood flow and pressure in the pulmonary artery. At the autopsy it appeared that all the circumstances were present, from which the diagnosis of this anomaly is usually made, except for the septal defect itself. East (1940) in describing his case of primary pulmonary hypertension, makes it clear that the same signs had made the same diagnosis plausible. It was therefore natural to consider what circumstances could have a similar effect upon the dynamics of the lesser circulation. The only comparable situation, in which blood from the right ventricle might pass through a peripheral channel to return to the right auricle and thus round again, would be if there were a communication between the pulmonary artery and a bronchial vein. This was purely hypothetical, but it was to investigate this possibility that sections from the hila were specially asked for, and in one of these the arteriovenous communication was found. The presence of a systemic artery, shown by serial sections to be contributing to the aneurysm, makes it necessary to abandon the original hypothesis. The only alternative is an anastomosis between bronchial artery and pulmonary artery or vein.

In the developing lung the early pulmonary capillary plexus is fed by ventral branches of the dorsal aorta and this is its only source of supply until the pulmonary artery develops later from the ventral root of the sixth branchial arch. When the pulmonary artery takes over, the aortic connections regress until at birth they are represented by the bronchial arteries only. This process is well described by Huntington (1919), who states " the origin and variations of the bronchial arteries and the presence of anastomoses or larger connecting channels between them and the pulmonary arteries, depends on the organization of the early postbranchial pulmonary plexus."

When the bronchial arteries were rediscovered by Ruysch in 1732, he assumed that they anastomosed with the pulmonary artery and this belief was maintained until fairly recent times. But it is now generally accepted that there is no direct arterial anastomosis in normal health. The experiments of W. S. Miller (1892-1925) using injection methods and of Berry and Daly (1931) using the heart-lung preparation, agree in demonstrating that the two arterial systems communicate only through the capillary bed to which both contribute. These experiments were done on the lungs of dogs in normal health. On the other hand, Rakshit (1949) has recently demonstrated vessels that connect the bronchial with the pulmonary artery in the lung hila of the guinea-pig and the rat.

It has long been known that in cases of congenital pulmonary atresia there is hypertrophy of the bronchial arteries with direct communications between them and the pulmonary arteries. Wood and M. Miller (1938) in an important paper describing the result of injections of dye into the aorta when all exits from the aorta were ligatured except the bronchial arteries, were able to demonstrate greatly increased communications between the bronchial and pulmonary arteries in certain diseases. These abnormal communications occurred generally in cases of mitral stenosis and locally in cases of primary bronchial carcinoma and in pulmonary tuberculosis. In the latter disease, when the subject had died after a hæmoptysis, the injection mass derived from the aorta was found in the cavity, suggesting that the fatal hæmorrhage had occurred from the bronchial artery. Most significantly to this paper, one of their 74 patients, diagnosed "Ayerza's disease" but from the description evidently an example of primary pulmonary hypertension, showed on injection of the aorta a complete outline of the pulmonary arterial tree. The vascular shunt between the two circulations was in this case greater than in any other and although no histological examination 
is mentioned, the underlying abnormality must have been something very like that found in the present case.

Cauldwell et al. (1948) confirm these observations as regards pulmonary tuberculosis, but there are few other reports as to the possibility of such shunts. Hovelacque et al. (1936) in an anatomical investigation of the bronchial arterial system, found in 6 of the 30 subjects they examined a direct extrapulmonary anastomosis between a main bronchial artery and a pulmonary vein. They illustrate one of these and certainly the contributing bronchial artery appears to be more than a mere vas vasorum: there is no note as to the cause of death in their cases. There is, too, no confirmation of this finding in the much more extensive work on the same subject by Cauldwell et al. (1948).

Taussig (1947) describes a case of Eisenmenger's complex in which " the pulmonary artery was enormously enlarged and there were remarkable changes in the lungs which resembled arteriovenous aneurysms." It is not likely that these were pulmonary arteriovenous communications of the usual sort for the patient was not cyanosed until the age of 16. Elsewhere, Taussig stresses the difficulty of distinguishing clinically between the Eisenmenger complex and "Ayerza's disease."

It is here suggested that primary pulmonary hypertension results from the excessive entry of blood into the lesser circulation from the bronchial arteries. This blood, being injected under systemic pressure causes dilatation and sclerotic changes in the pulmonary artery and hypertrophy with eventual failure of the right ventricle. In the case under review the abnormal communication may have been a developmental abnormality, a failure of regression of the ventral branches of the dorsal aorta within the lung. Such an anastomosis, established potentially before birth, would tend in the course of years to carry increasing quantities of blood, and thus eventually to cause death from right ventricular failure.

It should be possible in future cases to test this hypothesis by post-mortem injection methods such as those of Wood and Miller (1938), followed by a search of the hila for anastomoses. If it be allowed that these anastomoses may occur spontaneously, then a method of treatment by ligation of the bronchial arteries may arise, in those cases that are not too far advanced to preclude a thoracotomy.

\section{SUMMARY}

A case of primary pulmonary hypertension is presented which showed paralysis of the left vocal cord.

At necropsy an arteriovenous communication was found; this was probably an anastomosis between bronchial artery and pulmonary artery.

The significance of this finding is discussed.

My thanks are due to Dr. W. N. Mann under whom the patient was admitted to Guy's Hospital, to Dr. S. J. De Navasquez for his help in the histology, and to Dr. Maurice Campbell for his general advice. I am also grateful to Brigadier Bennett, Consultant Physician to the Army, for permitting me to see the patient's previous case-notes.

\section{REFERENCES}

Berry, J. L., and Daly, I. de B. (1931). Proc. Roy. Soc., London, 109, 319.

Brenner, O. (1935). Arch. intern. Med., 56, 976.

Cauldwell, E. W., Sickert, R. G., Lininger, R. E., Anson, B. J. (1948). Surg. Gynaec. and Obstet., 86, 395.

De Navasquez, S. J., Forbes, J. R., and Holling, H. E. (1940). Brit. Heart J., 2, 177.

Dolowitz, D. A., and Lewis, C. S. (1948). Amer. J. Med., 4, 856.

Erlanger, H., and Levine, S. A. (1943). Amer. Heart J., 26, 520.

Harrison, C. V. (1948). J. Path. Bact., 60, 289.

Hovelacque, A., Monod, O. and Evrard, H. (1936). Ann. anat. path., Paris, 13, 129.

Huntington, G. S. (1919). Anat. Rec., 17, 165.

Kraus (1901), quoted by Fetterolf, G., and Norris, G. W. (1911). Amer. J. med. Sci., 141, 625.

Lewis, T. (1914). Heart, 5, 367.

Miller, W. S. (1937). The Lung, Springfield, Illinois.

Rakshit, P. (1939). Quart. J. exp. Physiol., 35, 47.

Ruysch, quoted by Miller, W. S. (1937).

Taussig (1947). Congenital Malformations of the Heart, New York, 1st ed., p. 416.

Wood, D. A., and Miller, M. (1938). J. Thor. Surg., 7, 649. 